NIST GCR 06-906

\title{
Probabilistic Models for Directionless Wind Speeds in Hurricanes
}

Mircea Grigoriu

Cornell University 


\title{
Probabilistic Models for Directionless Wind Speeds in Hurricanes
}

\author{
Prepared for \\ U.S. Department of Commerce \\ Building and Fire Research Laboratory \\ National Institute of Standards and Technology \\ Gaithersburg, MD 20899-8611
}

By

Mircea Grigoriu

Cornell University

Ithaca, NY 14853

December 2006

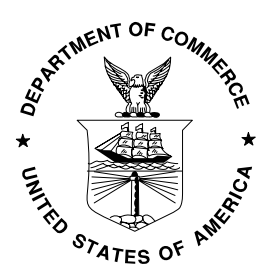

U.S. Department of Commerce Carlos M. Gutierrez, Secretary

Technology Administration Robert Cresanti, Under Secretary of Commerce for Technology 


\section{Contents}

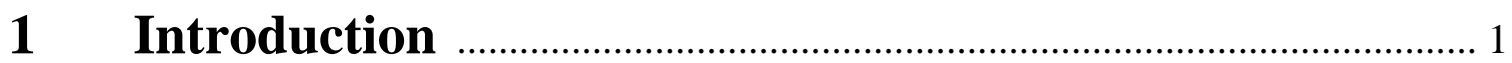

2 Probabilistic models for hurricanes ……………………......... 1

2.1 Hurricane time model and design wind speeds ............................1

2.2 Hurricane intensity. Gumbel distribution ...................................2

2.3 Hurricane intensity. Shifted Gamma distribution .......................

2.4 Hurricane intensity. Reverse Weibull distribution ....................4

2.5 Hurricane intensity. Generalized Pareto distribution ................5

2.5.1 The method of moments ....................................................................... 6

2.5.2 The method of probability weighted moments ................................. 6

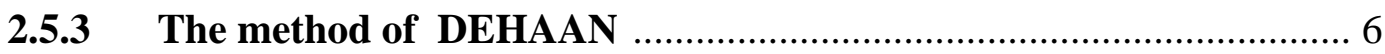

$3 \quad$ Monte Carlo algorithm ................................................................ 7

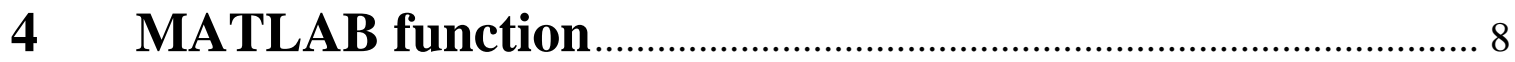

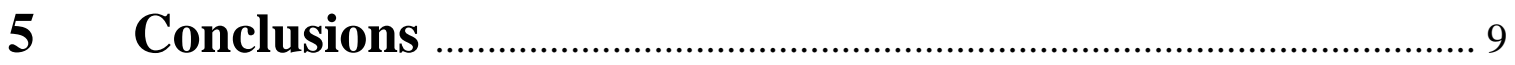

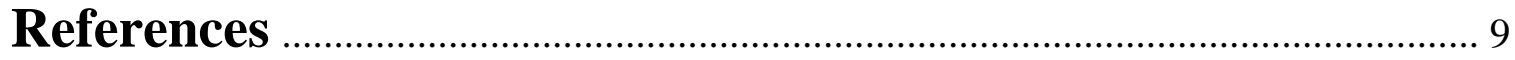

Appendix: MATLAB function hurr_nd_mc.m …………………....10 


\title{
Probabilistic models for directionless wind speeds in hurricanes
}

\author{
Mircea Grigoriu \\ Cornell University, Ithaca, NY 14853
}

\section{Introduction}

Extensive records of simulated hurricane wind speeds for 16 azimuth directions are available at, for example, the NIST web site http://www.nist.gov/wind. These records cover time periods of the order 2,000 years so that may not be sufficient for estimating wind speeds with average return periods of the order 1,000 years. Probabilistic models are needed to predict extreme wind speeds corresponding to such return periods.

Our objectives are to (1) develop probabilistic models for hurricane wind speeds recorded irrespective of direction, referred to here as directionless wind speeds, (2) calibrate these models to wind records, and (3) provide a Monte Carlo algorithm for generating data sets over long time periods that are consistent with a site statistics. Four models are considered for extreme wind speeds, the Gumbel, the shifted Gamma, the reverse Weibull, and the Pareto distributions. The study uses extreme wind speeds irrespective of direction, referred to as directionless wind speeds.

\section{Probabilistic models for hurricanes}

Hurricanes arrive at a site at random times and are characterized by random extreme wind speeds. Records can be used to estimate the mean number $\nu$ of hurricanes per year at a site. Records can also be used to construct the distribution $F$ of extreme wind speeds under the assumption that wind speeds recorded in different hurricanes belong to the same statistical population, that is, they are independent samples of $F$.

\subsection{Hurricane time model and design wind speeds}

Let $T_{1}<T_{2}<\cdots$ be a random sequence denoting the arrival times of hurricanes at a site, and let $X_{1}, X_{2}, \ldots$ be hurricane wind speeds irrespective of direction.

It is assumed that (1) the random sequences $T_{1}, T_{2}, \ldots$ and $X_{1}, X_{2}, \ldots$ are mutually independent, (2) the random variables $X_{1}, X_{2}, \ldots$ are independent and have the same distribution $F$, (3) the hurricane season begins on June 1 and ends November 30, and (3) the random variables $T_{1}, T_{2}-T_{1}, \ldots$ are independent and follow an exponential distribution with 
mean $1 / \nu, \nu>0$, so that $T_{1}, T_{2}, \ldots$ are points of a Poisson process $N$ with intensity $\nu$. The Poisson process $N$ is on only during the hurricane season.

Consider a time interval $[0, \tau], \tau>0$, and note that $N(\tau)$ gives the random number of hurricanes in $[0, \tau]$. The distribution $F_{\tau}$ of the largest extreme wind speed in $[0, \tau]$, that is, the random variable $\max _{1 \leq i \leq N(\tau)}\left\{X_{i}\right\}$, is

$$
F_{\tau}(x)=\exp [-\nu \tau(1-F(x))]
$$

since the probability of $n$ hurricanes occurring in $[0, \tau]$ is $P(N(\tau)=n)=(\nu \tau)^{n} \exp (-\nu \tau) / n$ ! and the probability that the conditional random variable $\max _{1 \leq i \leq N(\tau)}\left\{X_{i}\right\} \mid(N(\tau)=n)$ does not exceed $x$ is equal to $F(x)^{n}$.

Suppose we retain from the sequence $X_{1}, X_{2}, \ldots$ of wind speeds at a site only those values exceeding a threshold $x$, so that the resulting process describes hurricanes with wind speeds larger than $x$. The mean arrival rate of these hurricanes is $\nu(x)=\nu(1-F(x))$, so that $1 / \nu(x)$ gives the average time between consecutive wind speeds larger than $x$. Hence, the design wind speed that is exceeded on average once in $r$ years, that is, the $r$-year wind speed, results by setting $1 / \nu(x)$ equal to $r$ and is

$$
x^{(r)}=F^{-1}\left(1-\frac{1}{\nu r}\right) \text {. }
$$

We consider four models for $F$, the Gumbel, the shifted Gamma, the reverse Weibull, and the generalized Pareto distributions. The method of moments and other methods are used in the following two subsections to estimate the parameters of these models from wind records. The uncertainty in the estimates of the parameters of $F$ and the corresponding $r$-year wind speeds is quantified in a subsequent section.

\subsection{Hurricane intensity. Gumbel distribution}

A random variable $X$ is said to a Gumbel or extreme type 1 variable with parameters $(\alpha, u)$ if it has the distribution

$$
F(x)=\exp [-\exp (-\alpha(x-u))], \quad-\infty<x<\infty,
$$

and density

$$
f(x)=\alpha \exp [-\alpha(x-u)-\exp (-\alpha(x-u))], \quad-\infty<x<\infty .
$$

We use the notation $X \sim E X 1(\alpha, u)$ to indicate that $X$ is a Gumbel variable with parameters $(\alpha, u)$. The parameters $(\alpha, u)$ are denoted by (al_gumbel, $\mathbf{u}_{-}$gumbel) in the MATLAB code hurr_nd_mc.m.

The mean $\mu$ and standard deviation $\sigma$ of $X$ are related to the parameters $(\alpha, u)$ by

$$
\begin{aligned}
& u=\mu-\frac{0.577216}{\alpha} \\
& \alpha=\frac{\pi}{\sigma \sqrt{6}} .
\end{aligned}
$$

These relationships with $\mu$ and $\sigma$ replaced by their estimates $\hat{\mu}$ and $\hat{\sigma}$ are used in hurr $\_$nd $\_\mathbf{m c} . \mathbf{m}$ to obtain estimates of $(\alpha, u)$. 


\subsection{Hurricane intensity. Shifted Gamma distribution}

Let $\tilde{X}$ be a Gamma random variable with parameters $(k, \lambda), k>0, \lambda>0$, and density

$$
f(x)=\frac{\lambda^{k} x^{k-1} e^{-\lambda x}}{\Gamma(k)}, \quad x \geq 0,
$$

where $\Gamma(\cdot)$ denotes the Gamma function. The distribution of $\tilde{X}$ is

$$
F(x)=\int_{0}^{x} f(u) d u=\int_{0}^{\lambda x} \frac{z^{k-1} e^{-z}}{\Gamma(k)} d z, \quad x \geq 0,
$$

and is given by, for example, the MATLAB function cdf('Gamma', $x, k, 1 / \lambda)$. The solution $x_{p}$ of $F\left(x_{p}\right)=p \in[0,1]$, that is, the $p$-fractile of $F$ can be obtained from the MATLAB function icdf('Gamma', $p, k, 1 / \lambda)$.

Consider the random variable $X=a+\tilde{X}$, where $a$ is a real constant. Since $P(X \leq$ $x)=P(\tilde{X} \leq x-a)$, the density and distribution of $X$ are given by Eqs. 6 and 7 with $x-a$ in place of $x$, and are valid for $x \geq a$. The mean $\mu$, variance $\sigma^{2}$, skewness $\gamma_{3}$, and kurtosis $\gamma_{4}$ of $X$ are

$$
\begin{aligned}
\mu & =a+k / \lambda \\
\sigma^{2} & =k / \lambda^{2} \\
\gamma_{3} & =2 / \sqrt{k} \\
\gamma_{4} & =3(1+2 / k) .
\end{aligned}
$$

Suppose that the sequence of extreme wind speeds $X_{1}, X_{2}, \ldots$ follows a shifted Gamma distribution and that a record $\left(x_{1}, \ldots, x_{n}\right)$ of this sequence is available. Our objective is to estimate the parameters $(a, k, \lambda)$ of this distribution. The maximum likelihood method and the method of moments are commonly used to calculate estimates $(\hat{a}, \hat{k}, \hat{\lambda})$ of $(a, k, \lambda)$. Maximum likelihood estimates have smaller variance that those obtained by the method of moments but can be unstable for some values of $k$ ([3], Section 7.1). To avoid such difficulties, the method of moments is used to estimate $(a, k, \lambda)$. Suppose that estimates $\left(\hat{\mu}, \hat{\sigma}, \hat{\gamma_{3}}, \hat{\gamma_{4}}\right)$ of the moments $\left(\mu, \sigma, \gamma_{3}, \gamma_{4}\right)$ of $X_{1}, X_{2}, \ldots$ have been calculated from the available record $\left(x_{1}, \ldots, x_{n}\right)$. Then $k$ can be estimated from the expression of the coefficient of skewness or kurtosis and the estimates of these coefficients. For example, $\hat{k}=4 / \hat{\gamma}_{3}{ }^{2}$ if the relationship between $k$ and $\gamma_{3}$ is used. Alternatively, $k$ can be determined from the relationship between $k$ and $\gamma_{4}$. The MATLAB code hurr_nd_mc.m uses the relationships

$$
\begin{aligned}
& \hat{k}=4 / \hat{\gamma}_{3}{ }^{2} \\
& \hat{\lambda}=\sqrt{\hat{k}} / \hat{\sigma}
\end{aligned}
$$

to find estimates of $k$ and $\lambda$, and calculates estimates $\hat{a}$ of the shift $a$ by two options. The first option sets $\hat{a}=\min _{1 \leq i \leq n}\left\{x_{i}\right\}$. The second option calculates $\hat{a}$ from $\hat{a}=\hat{\mu}-\hat{k} / \hat{\lambda}$. If the resulting estimate of $a$ is such that $\hat{a}>\min _{1 \leq i \leq n}\left\{x_{i}\right\}$, we set $\hat{a}=\min _{1 \leq i \leq n}\left\{x_{i}\right\}$. The parameters $(a, k, \lambda)$ are denoted in hurr_nd_mc.m by (shift1, kq1, lamq1) and (shift2, kq2, lamq2) for options 1 and 2, respectively. 


\subsection{Hurricane intensity. Reverse Weibull distribution}

Let $Y$ be a Weibull random variable with parameters $\alpha>0, \xi \in \mathbb{R}$, and $c>0$, distribution

$$
F(y)= \begin{cases}1-\exp \left[-\left(\frac{y-\xi}{\alpha}\right)^{c}\right], & y>\xi \\ 0 & y \leq \xi\end{cases}
$$

and density

$$
f(y)=\frac{c}{\alpha}\left(\frac{y-\xi}{\alpha}\right)^{c-1} \exp \left[-\left(\frac{y-\xi}{\alpha}\right)^{c}\right], \quad y>\xi .
$$

Values of distribution $F(y)$ and solutions of $F\left(y_{p}\right)=p \in[0,1]$ can be calculated by, for example, the MATLAB functions $F(y)=\operatorname{cdf}($ 'wbl', $y-\xi, \alpha, c)$ and $y_{p}-\xi=\operatorname{icdf}($ 'wbl', $p, \alpha, c)$.

Moments of any order of $Y$ can be obtained from moments $E\left[\tilde{Y}^{q}\right]=\Gamma(1+q / c)$ of the scaled random variable $\tilde{Y}$ defined by $Y=\xi+\alpha \tilde{Y}$ ([3], Chapter 20). For example, the mean $\mu_{y}$, variance $\sigma_{y}^{2}$, and skewness $\gamma_{y, 3}$ of $Y$ are

$$
\begin{aligned}
\mu_{y} & =\xi+\alpha \Gamma(1+1 / c) \\
\sigma_{y}^{2} & =\alpha^{2}\left(\Gamma(1+2 / c)-\Gamma(1+1 / c)^{2}\right) \\
\gamma_{y, 3} & =\frac{\Gamma(1+3 / c)-3 \Gamma(1+1 / c) \Gamma(1+2 / c)+2 \Gamma(1+1 / c)^{3}}{\left(\Gamma(1+2 / c)-\Gamma(1+1 / c)^{2}\right)^{3 / 2}} .
\end{aligned}
$$

The properties of the random variable $X \stackrel{d}{=}-Y$, called reverse Weibull variable, result from those of $Y$. For example, the distribution of $X$, that is, the probability $P(X \leq x)=$ $P(Y>-x)$, is equal to $\exp \left[-\left(\frac{\eta-x}{\alpha}\right)^{c}\right]$ for $x<\eta=-\xi$ and 1 for $x \geq \eta$.

Suppose that the sequence of extreme wind speeds $X_{1}, X_{2}, \ldots$ follows a reverse Weibull distribution and that a record $\left(x_{1}, \ldots, x_{n}\right)$ of this sequence is available. Our objective is to estimate the parameters $(\alpha, \eta=-\xi, c)$ of the reverse Weibull distribution. The method of moments, the method of maximum likelihood, the method of probability-weighted moments, and other methods can be used to estimate the parameters of this distribution ([4], Chapter 22). Extensive numerical studies suggest that the method of moments delivers satisfactory estimators for the unknown parameters of $F$, in contrast to, for example, the maximum likelihood method that can produce unstable estimators [6]. These features of the method of moments and its simplicity are the reasons for selecting the method for our analysis. The following 3 step algorithm can be used to estimate the parameters $(\alpha, \eta=-\xi, c)$ by the method of moments.

Step 1. Construct the record $\left(y_{1}=-x_{1}, \ldots, y_{n}=-x_{n}\right)$. Since the record $\left(x_{1}, \ldots, x_{n}\right)$ is assumed to consist of independent samples of a reverse Weibull distribution with parameters $(\alpha, \eta=-\xi, c)$, the record $\left(y_{1}, \ldots, y_{n}\right)$ consists of independent samples of a Weibull distribution with parameters $(\alpha, \xi, c)$.

Step 2. Calculate estimates $\hat{\mu}_{y}, \hat{\sigma}_{y}^{2}$, and $\hat{\gamma}_{y, 3}$ for the mean $\mu_{y}$, variance $\sigma_{y}^{2}$, and skewness coefficient $\gamma_{y, 3}$ from the sample $\left(y_{1}, \ldots, y_{n}\right)$. For example, $\hat{\mu}_{y}=\sum_{i=1}^{n} y_{i} / n, \hat{m}_{q}=$ $\sum_{i=1}^{n}\left(y_{i}-\hat{\mu}_{y}\right)^{q} / n$ are estimates of the central moments of order $q \geq 2, \hat{\sigma}_{y}^{2}=\hat{m}_{2}$, and $\hat{\gamma}_{q}=\hat{m}_{q} / \hat{\sigma}_{y}^{q / 2}$ for $q=3,4$. 
Step 3. Estimates the parameters $(\alpha, \xi, c)$ from Eq. 12. First, find an estimate $\hat{c}$ for $c$ from the last equality in Eq. 12 with $\hat{\gamma}_{y, 3}$ in place of $\gamma_{y, 3}$. This nonlinear equation needs to be solved by iterations. Second, find an estimate $\hat{\alpha}$ for $\alpha$ from the second equality in Eq. 12 with $\left(\sigma_{y}^{2}, c\right)$ replaced by $\left(\hat{\sigma}_{y}^{2}, \hat{c}\right)$. Third, find an estimate $\hat{\xi}$ for $\xi$ from the first equality in Eq. 12 with $\left(\mu_{y}, \alpha, c\right)$ replaced by $\left(\hat{\mu}_{y}, \hat{\alpha}, \hat{c}\right)$. If $\hat{\xi}>\min _{1 \leq i \leq n}\left\{y_{i}\right\}$, then set $\hat{\xi}=\min _{1 \leq i \leq n}\left\{y_{i}\right\}$ and calculate $(\hat{\alpha}, \hat{c})$ from the first equalities in Eq. 12 with $\left(\hat{\mu}_{y}, \hat{\sigma}_{y}^{2}\right)$ in place of $\left(\mu_{y}, \sigma_{y}^{2}\right)$.

The estimates $(\hat{\alpha}, \hat{\xi}, \hat{c})$ of the parameters $(\alpha, \xi, c)$ delivered by the above algorithm are denoted in hurr_nd_mc.m by (alw, xiw, cw).

\subsection{Hurricane intensity. Generalized Pareto distribution}

Let $X$ be a (maximal) generalized Pareto variable with distribution

$$
F(x)= \begin{cases}1-(1-k x / \alpha)^{1 / k}, & 1-k x / \alpha \geq 0, k \neq 0, \alpha>0 \\ 1-\exp (-x / \alpha), & x \geq 0, k=0, \alpha>0\end{cases}
$$

and density

$$
f(x)= \begin{cases}(1 / \alpha)(1-k x / \alpha)^{1 / k}, & 1-k x / \alpha \geq 0, k \neq 0, \alpha>0 \\ (1 / \alpha) \exp (-x / \alpha), & x \geq 0, k=0, \alpha>0\end{cases}
$$

depending on the scale and shape parameters $\alpha$ and $k$, respectively. The range of the argument $x$ of $F$ is $[0, \infty)$ and $[0, \alpha / k]$ for $k \leq 0$ and $k>0$, respectively. We denote the distribution $F$ in Eq. 13 by $G P D(\alpha, k)$. The parameters $(\alpha, k)$ of $F$ and the mean $\mu$ and variance $\sigma^{2}$ of this distribution are related by

$$
\begin{aligned}
\alpha & =\frac{\mu}{2}\left(\mu^{2} / \sigma^{2}+1\right) \\
k & =\frac{1}{2}\left(\mu^{2} / \sigma^{2}-1\right) .
\end{aligned}
$$

The $p$-fractile $x_{p}$ of $F \sim G P D(\alpha, k)$, that is, the solution of $F\left(x_{p}\right)=p$, has the expression

$$
x_{p}= \begin{cases}\alpha\left(1-(1-p)^{k}\right) / k, & k \neq 0 \\ -\alpha \ln (1-p), & k=0\end{cases}
$$

A notable property of generalized Pareto random variables is that, if $X$ follow a $G P D(\alpha, k)$ distribution, then the conditional random variable $(X-a) \mid(X>a)$ is $G P D(\alpha-$ $k a, k)$ for any $a \in \mathbb{R}$. This invariance property simplify significantly the construction and analysis of peaks over threshold sequences associated with generalized Pareto series.

Suppose that a record $\left(x_{1}, \ldots, x_{n}\right)$ of independent values of $X$ with distribution $F$ in Eq. 13 is available. Our objective is to estimate the parameters $(\alpha, k)$ of $F$ from the record $\left(x_{1}, \ldots, x_{n}\right)$. The maximum likelihood, probability-weighted moments, moments, and other methods can be used to estimate the parameters of $F$ ([1], Section 10.3 and 10.8). Extensive simulation studies indicate that estimates of $(\alpha, k)$ delivered by the method of moments are 
generally reliable unless $k<-0.2$ and that the method of probability-weighted moments is adequate for $k<0$ [2]. Other studies found the method of moments to be satisfactory for a broader range of values of $k$ [5]. The MATLAB code hurr_nd_mc.m uses three methods for estimating the parameters $(\alpha, k)$ from data, the method of moments, the method of probability weighted moments, and the method of DEHAAN.

\subsubsection{The method of moments}

Estimates of the parameters $(\alpha, k)$ can be calculated from

$$
\begin{aligned}
\hat{k} & =\frac{1}{2}\left(\bar{x}^{2} / s^{2}-1\right) \\
\hat{\alpha} & =\frac{\bar{x}\left(\bar{x} / s^{2}+1\right)}{2},
\end{aligned}
$$

where $\bar{x}=\sum_{i=1}^{n} x_{i} / n$ and $s^{2}=\sum_{i=1}^{n}\left(x_{i}-\bar{x}\right) / n$ denote the sample mean and variance of $\left(x_{1}, \ldots, x_{n}\right)$, respectively.

Suppose we construct from the original record $\left(x_{1}, \ldots, x_{n}\right)$ a new record $\left(y_{1}, \ldots, y_{m}\right)$, $m \leq n$, consisting only of those readings $x_{i}$ exceeding a specified threshold $a$. The relationships in Eq. 17 with $\left(m,\left\{z_{j}=y_{j}-a\right\}\right)$ in place of $\left(n,\left\{x_{i}\right\}\right)$ can be used to estimate the parameters of the Pareto variable $Y-a$.

\subsubsection{The method of probability weighted moments}

Let $\left\{x_{i: n}\right\}$ be the data set $\left\{x_{i}\right\}$ sorted in increasing order, that is, $x_{1: n} \leq x_{2: n} \leq \cdots \leq$ $x_{n: n}$. Set $p_{i: n}=(i-0.35 / n), i=1, \ldots, n$, and $t=(1 / n) \sum_{i=1}^{n}\left(1-p_{i: n}\right) x_{i: n}$. The the estimates of $\alpha$ and $k$ are given by ([1], Section 10.8.3)

$$
\begin{aligned}
\hat{k} & =\frac{t-\bar{x}}{\bar{x}-2 t} \\
\hat{\lambda} & =\frac{2 \bar{x} t}{\bar{x}-2 t} .
\end{aligned}
$$

Similar estimates can be constructed for a record $\left(y_{1}, \ldots, y_{m}\right), m \leq n$, consisting of values of $\left(x_{1}, \ldots, x_{n}\right)$ above a threshold $a$.

\subsubsection{The method of DEHAAN}

Let $\left(y_{1}, \ldots, y_{m}\right), m \leq n$, a record consisting of values of $\left(x_{1}, \ldots, x_{n}\right)$ above a threshold $a$. Denote by $\left\{y_{j: m}\right\}$ the data set $\left\{y_{j}\right\}$ sorted in increasing order, that is, $y_{1: m} \leq y_{2: m} \leq \cdots \leq$ $y_{m: m}$, and consider the statistics

$$
\mu_{n}^{(r)}=\frac{1}{m-1} \sum_{j=2}^{m-1}\left[\ln \left(y_{j+1: m}\right)-\ln \left(y_{1: m}\right)\right]^{r}, \quad r=1,2, \ldots
$$


for some $q \leq n$. The DEHAAN estimates of $\alpha$ and $k$ are defined in the NIST web site http://www.nist.gov/wind and are given by

$$
\begin{aligned}
& \hat{k}=-\mu_{n}^{(1)}-1+\frac{1 / 2}{1-\left(\mu^{(1)}\right)^{2} / \mu^{(2)}} \\
& \hat{\alpha}=a \mu_{n}^{(1)} / \rho,
\end{aligned}
$$

where $\rho=1$ for $\hat{k} \leq 0$ and $\rho=1 /(1-\hat{k})$ for $\hat{k}>0$.

The estimates of the parameters $(\alpha, k)$ delivered by the methods of moments, probability weighted moments, and DEHAAN are denoted in hurr_nd_mc.m by (al_pareto1, k_pareto1), (al_pareto2, k_pareto2), and (al_pareto3, k_pareto3), respectively. The threshold below which wind speed readings are disregarded is denoted by a_pareto for all estimation methods.

\section{Monte Carlo algorithm}

The hurricane hazard at a site during a time interval $[0, \tau]$ is completely specified by

- The arrival times $0=T_{0}<T_{1}<T_{2}<\cdots<T_{N(\tau)}$ of hurricanes and

- The extreme speeds $X_{1}, X_{2}, \ldots, X_{N(\tau)}$ recorded during each hurricane.

We have assumed that (1) the sequences $\left\{T_{i}\right\}$ and $\left\{X_{i}\right\}$ are independent of each other, (2) the arrival times $\left\{T_{i}\right\}$ define a Poisson process $N$ with intensity $\nu$ hurricanes/year, and (3) the random variables $X_{1}, X_{2}, \ldots$ are independent and follow either a reverse Weibull distribution or a shifted Gamma distribution.

A two-step Monte Carlo algorithm has been developed to generate samples of extreme wind speeds recorded during hurricane at a site. The input to the algorithm consists of a reference time interval $[0, \tau]$, the hurricane mean arrival rate $\nu$, the distribution shape $F$ of wind speeds $X_{1}, X_{2}, \ldots$, and the parameters of this distribution.

Step 1. Generate a sample $0=T_{0}<T_{1}<T_{2}<\cdots<T_{N(\tau)}$ of hurricane arrival times in $[0, \tau]$. As previously stated, it is assumed that hurricane can only occur from June 1 to November 30. Denote this time interval by $\tau_{y}$. The generation of hurricane arrival times can be based on the observation that the inter-arrival times $T_{k}-T_{k-1}, k=1, \ldots, N(\tau)$ are independent exponential random variables with mean $1 / \nu$. Hence, the time intervals $T_{k}-T_{k-1}$ are equal in distribution with the random variables $-\ln \left(U_{k}\right) / \nu$, where $U_{k}$ are independent random variables uniformly distributed in $[0,1]$. The MATLAB function rand can be used to generate samples of $U_{k}$. First, we generate a sample of $T_{1}=-\ln \left(U_{1}\right) / \nu$. If this sample is larger than $\tau_{y}$, there will be no hurricane at the site in this sample during a yearly hurricane season so that $N\left(\tau_{y}\right)=0$. Otherwise, the sample of $T_{1}$ gives the time of the first hurricane and $N\left(\tau_{y}\right) \geq 1$. Second, we generate a sample of $T_{2}-T_{1}=-\ln \left(U_{2}\right) / \nu$. If the sample of $T_{2}=\left(T_{2}-T_{1}\right)+T_{1}$ is larger than $\tau_{y}$ then $N\left(\tau_{y}\right)=1$. Otherwise, $N\left(\tau_{y}\right) \geq 2$ and we generate a sample of the following inter-arrival time. This process ends when an 
arrival time exceeds $\tau_{y}$ for the first time. A hurricane sample in $[0, \tau]$ consists of the sequence of hurricanes generated in each hurricane season of $[0, \tau]$. Denote by $N(\tau)$ the number of hurricanes in $[0, \tau]$. A similar procedure applies for the arrival times of hurricanes with wind speeds larger than a threshold $a_{\mathrm{thr}}$. The only difference is that the mean rate $\nu$ is replaced with the mean rate $\nu_{p}$ equal to $\nu$ scaled by the ratio $\#\left\{\right.$ hurricanes with speed $\left.\geq a_{\mathrm{thr}}\right\} / \#\{$ all hurricanes $\}$.

Step 2. Generate a sample $\boldsymbol{x}=\left(x_{1}, x_{2}, \ldots, x_{N(\tau)}\right)$ of the wind speeds $X_{1}, X_{2}, \ldots, X_{N(\tau)}$ corresponding to the sample of $0=T_{0}<T_{1}<T_{2}<\cdots<T_{N(\tau)}$ generated in the previous step. Let $\boldsymbol{u}=\left(u_{1}, u_{2}, \ldots, u_{N(\tau)}\right)$ be independent samples of a uniformly distributed random variable in $[0,1]$, which can be produced by, for example, the MATLAB function $\boldsymbol{u}=\operatorname{rand}(1, N(\tau))$. MATLAB functions have also been used to generate samples $\boldsymbol{x}$ of directionless hurricane wind speeds from $\boldsymbol{u}$ under various assumptions on the distribution of wind speed. For example, the sample $\boldsymbol{x}$ is given by $\boldsymbol{x}=-\left(\xi+\operatorname{icdf}\left({ }^{\prime} \mathrm{wbl}^{\prime}, \boldsymbol{u}, \alpha, c\right)\right)$ for the reverse Weibull distribution.

\section{MATLAB function}

A MATLAB function hurr_nd_mc.m has been developed for estimating the parameters of the Gumbel, shifted Gamma, reverse Weibull, and generalized Pareto distribution from hurricane wind speed records.

The input consists of:

(1) A record at a specified milepost (see lines 50-56),

(2) A range [cmin, cmax] for possible values of the tail parameter $c$ in Eqs. 10-11 of the reverse Weibull distribution and the number nc of intervals to be considered in this range,

(3) A threshold a_pareto for used for the generalized Pareto distribution,

(4) A number nyr of years selected for Monte Carlo simulation and a seed nseed for generating hurricane wind speeds, and

(5) A specified tail parameter cws for the reverse Weibull distribution.

The output consists of:

(1) A vector thurr with entries counting the number of hurricane in nyr years,

(2) Vectors of simulated hurricane wind speeds: x_gumbel_mc corresponding to the Gumbel distribution; x_shg1_mc and x_shg2_mc corresponding to the shifted Gamma distribution under option 1 and option 2; $\mathrm{x} \_$rw_mc corresponding to the reverse Weibull distribution; and x_par1_mc, x_par2_mc, and x_par2_mc corresponding to the generalized Pareto distribution with parameters estimated by the methods of moments, probability weighted moments, and DEHAAN. 
(3) Plots showing (i) histograms of the input wind record and Gumbel, shifted Gamma, reverse Weibull, and generalized Pareto densities fitted to this record, (ii) wind speeds of average return periods in the range $[50,1000]$ years predicted by the Gumbel, shifted Gamma, reverse Weibull, and generalized Pareto distributions fitted to the record using estimated parameters of these distributions and similar wind speeds derived directly from data, and (iii) wind speeds of average return periods in the range $[50,1000]$ years predicted by the reverse Weibull distribution with estimated and imposed tail parameter and similar wind speeds derived directly from data.

\section{Conclusions}

A MATLAB code has been developed for estimating parameters of the hurricane wind speeds described by Gumbel, shifted Gamma, reverse Weibull, and generalized Pareto distributions. Several estimation methods have been applied to calibrate these distributions to wind speeds records obtained from the NIST site http://www.nist.gov/wind. The resulting models have been used to generate synthetic hurricane wind speeds and estimate wind speeds with return periods in the range $[50,1000]$ years. The code produces numerous figures showing data and model statistics.

\section{References}

[1] E. Castillo, A. S. Hadi, N. Balakrishnan, and J. M. Sarabia. Extreme Value and Related Models with Applications in Engineering and Science. Wiley Series in Probability and Statistics, Hoboken, New Jersey.

[2] J. R. M. Hosking and J. R. Wallis. Parameter and quantile estimation for the generalized pareto dsitribution. Technometrics, 29(3):339-349, 1987.

[3] N. Johnson and S. Kotz. Distributions in Statistics: Continuous Univariate Distributions1. Houghton Mifflin Company, Boston, MA., 1970.

[4] N. L. Johnson, S. Kotz, and N. Balakrishnan. Continuous Univariate Distributions, volume 2. John Wiley \& Sons, Inc., New York, 1994. Second Edition.

[5] E. S. Martins and J. R. Stedinger. Generalized maximum likelihood pareto-poisson estimators for partial duration series. Water Resources Research, 37(10):2551-2557, 2001.

[6] Martins E. S. and J. R. Stedinger. Generalized maximum-likelihood generalized extemevalue quantile estimators for hydrological data. Water Resources Research, 36(3):737$744,2000$. 


\section{Appendix. MATLAB function hurr_nd_mc.m}

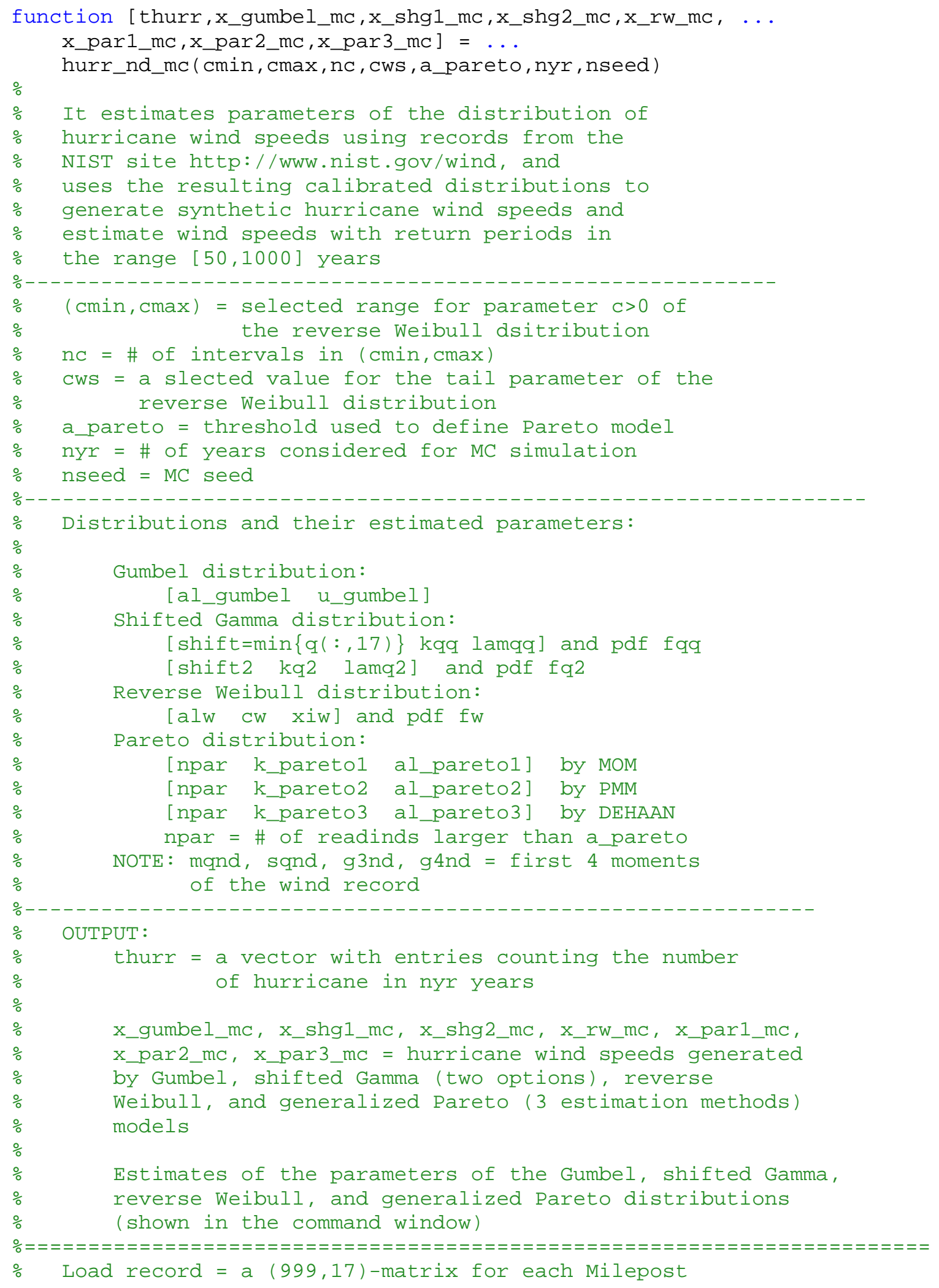




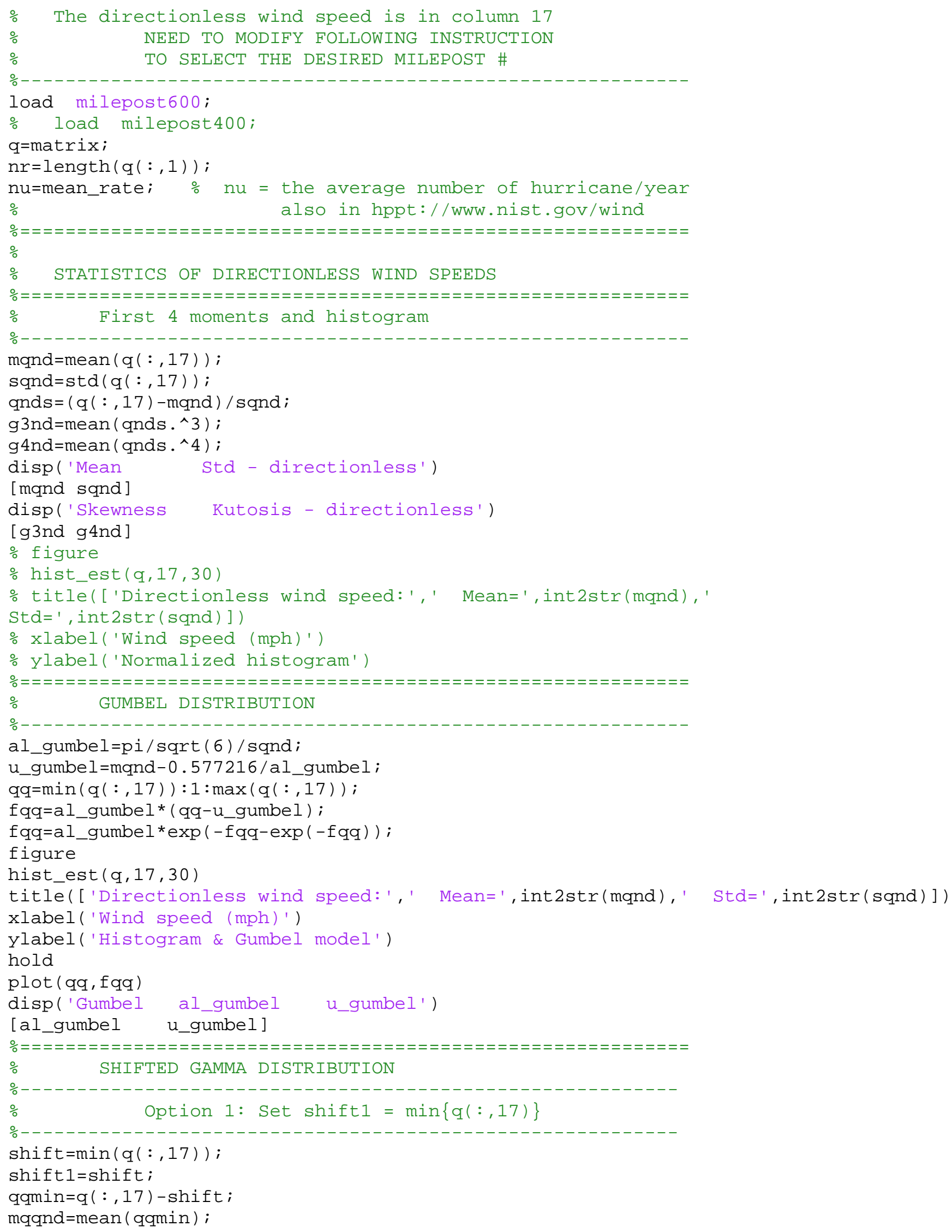




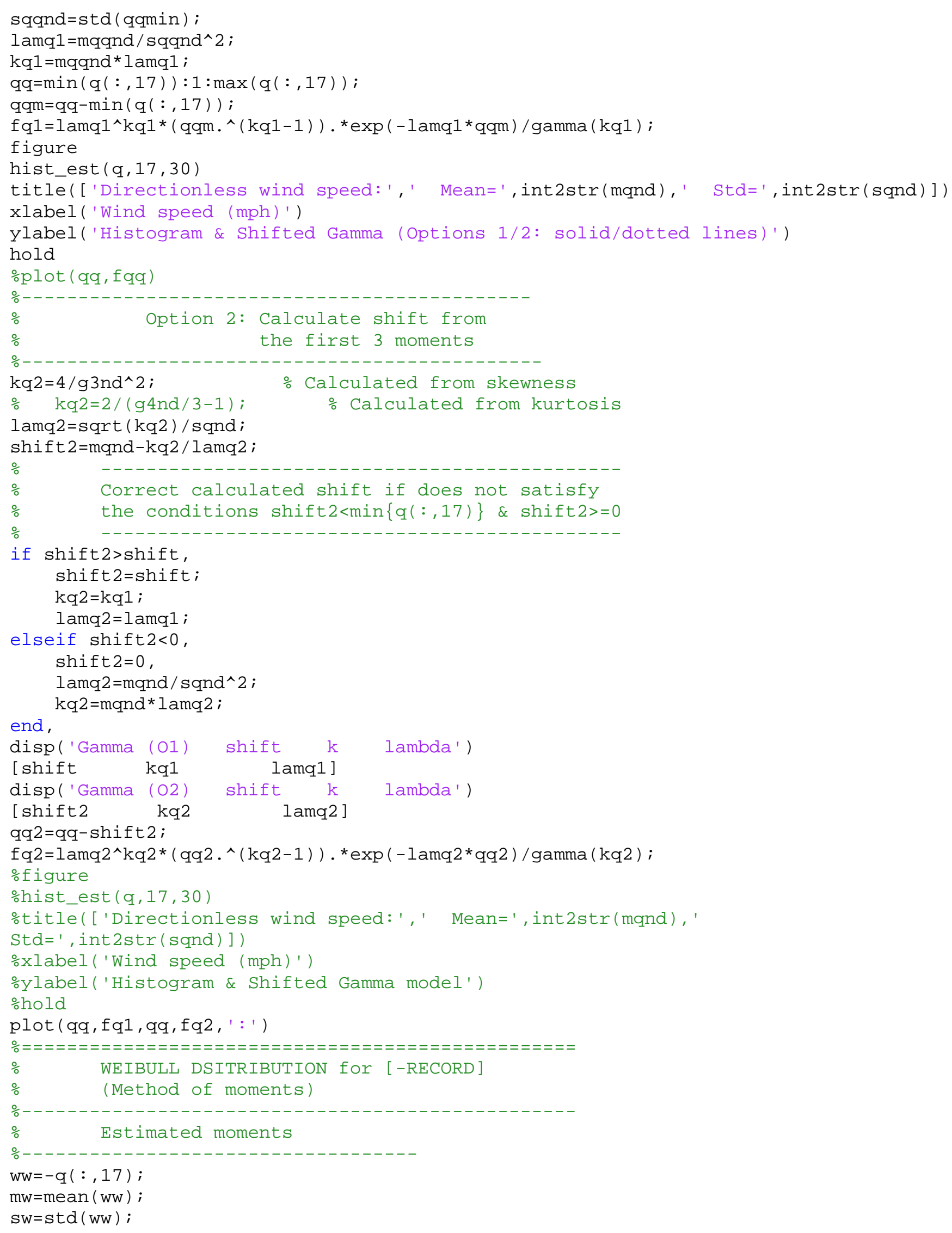




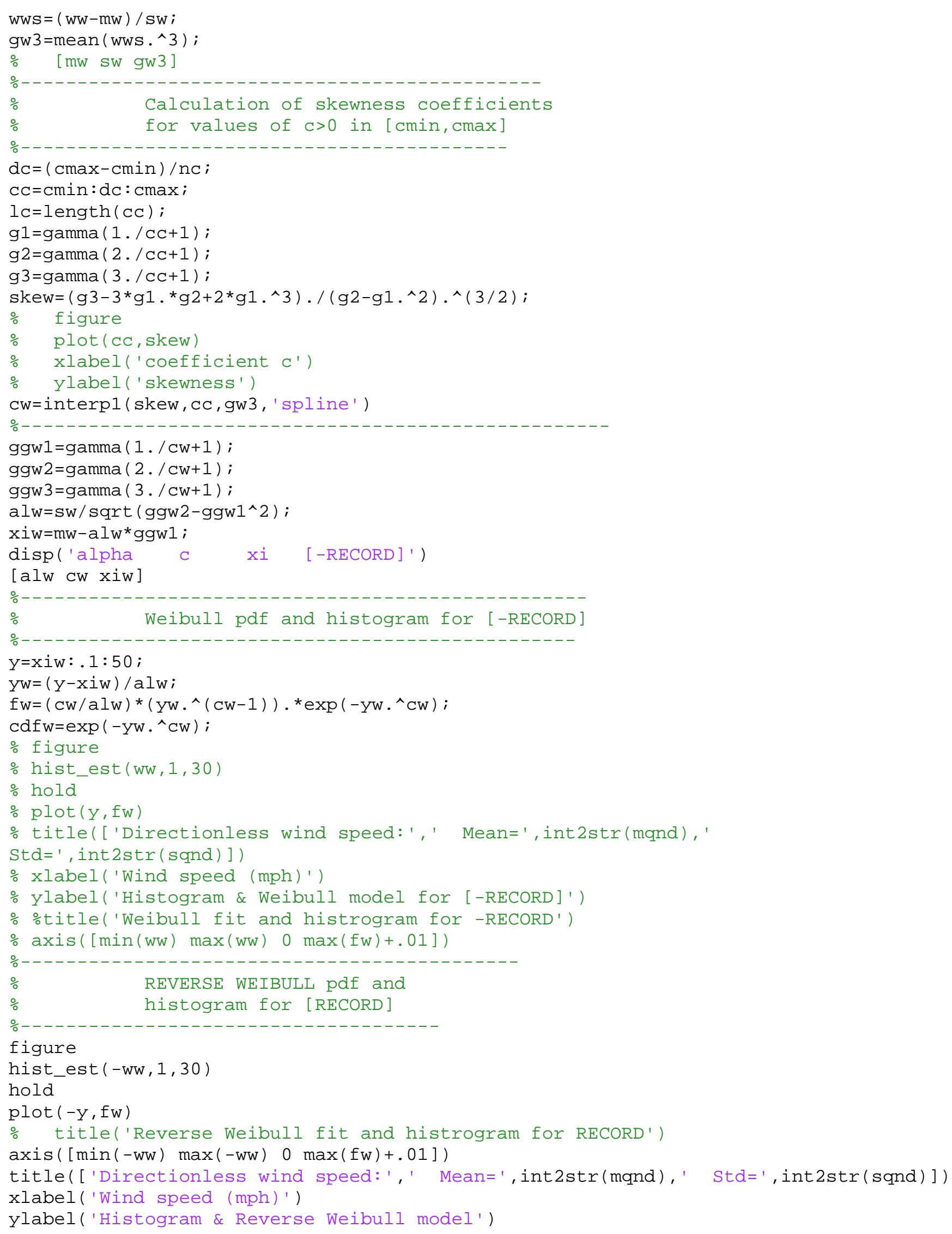




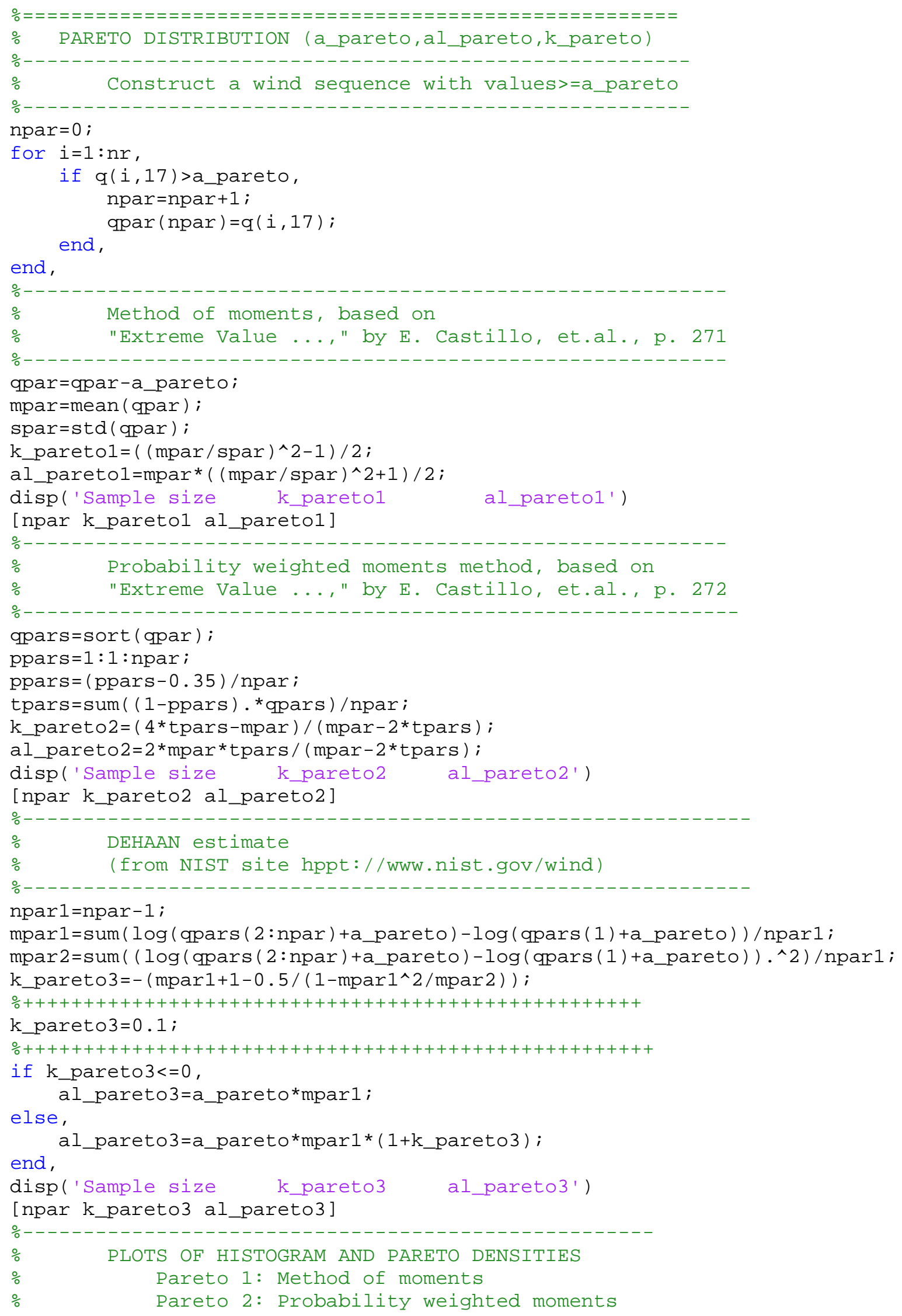




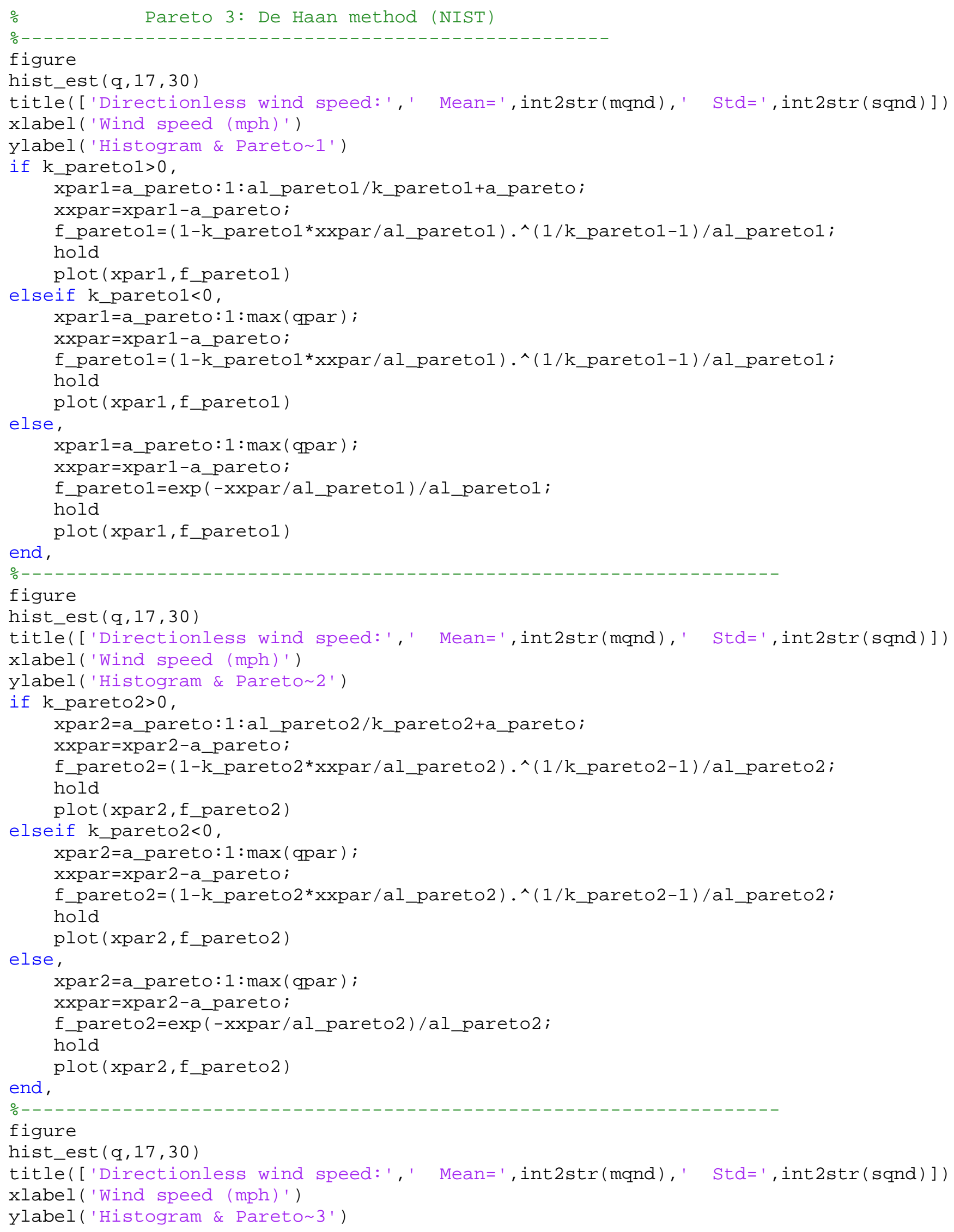




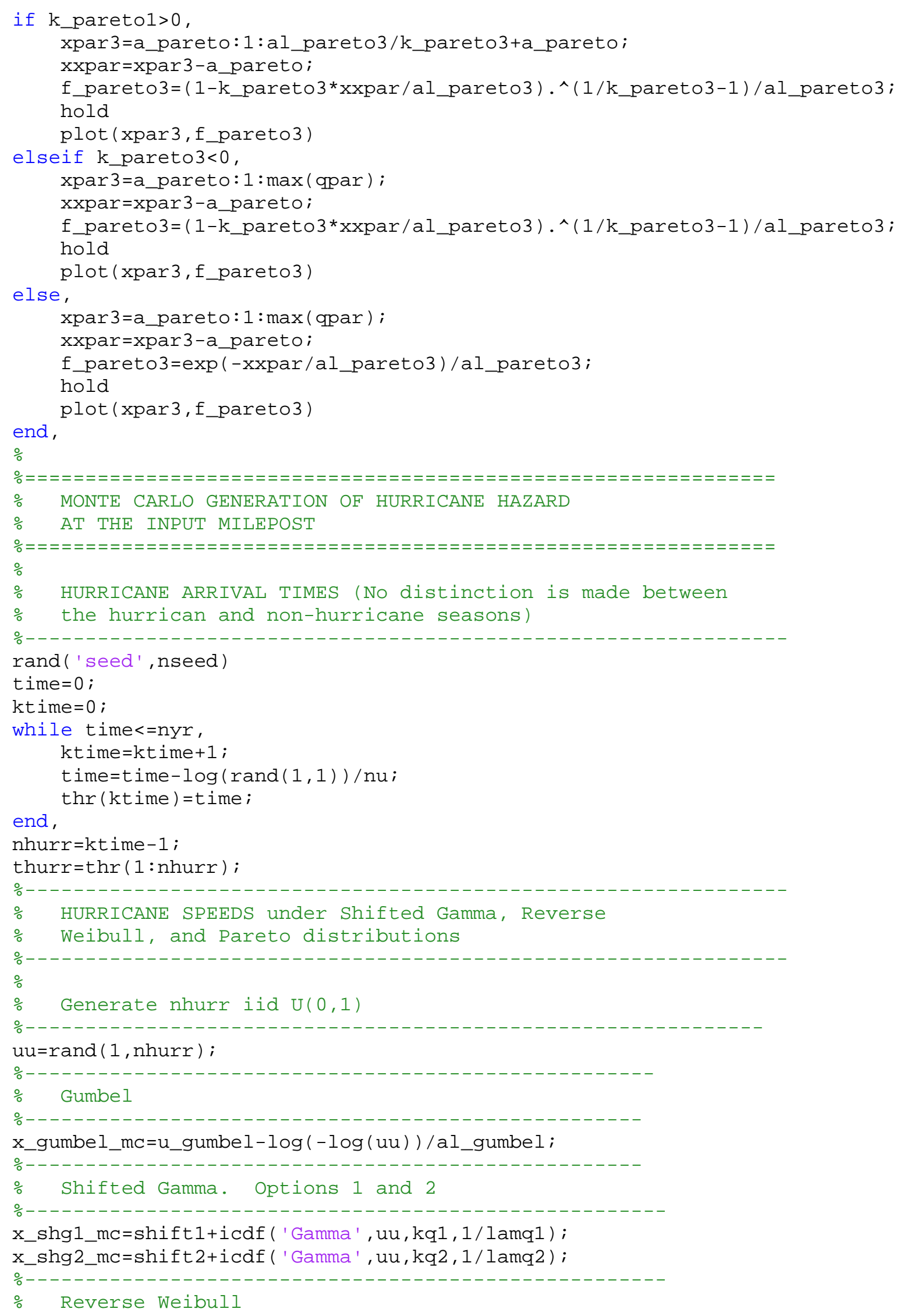




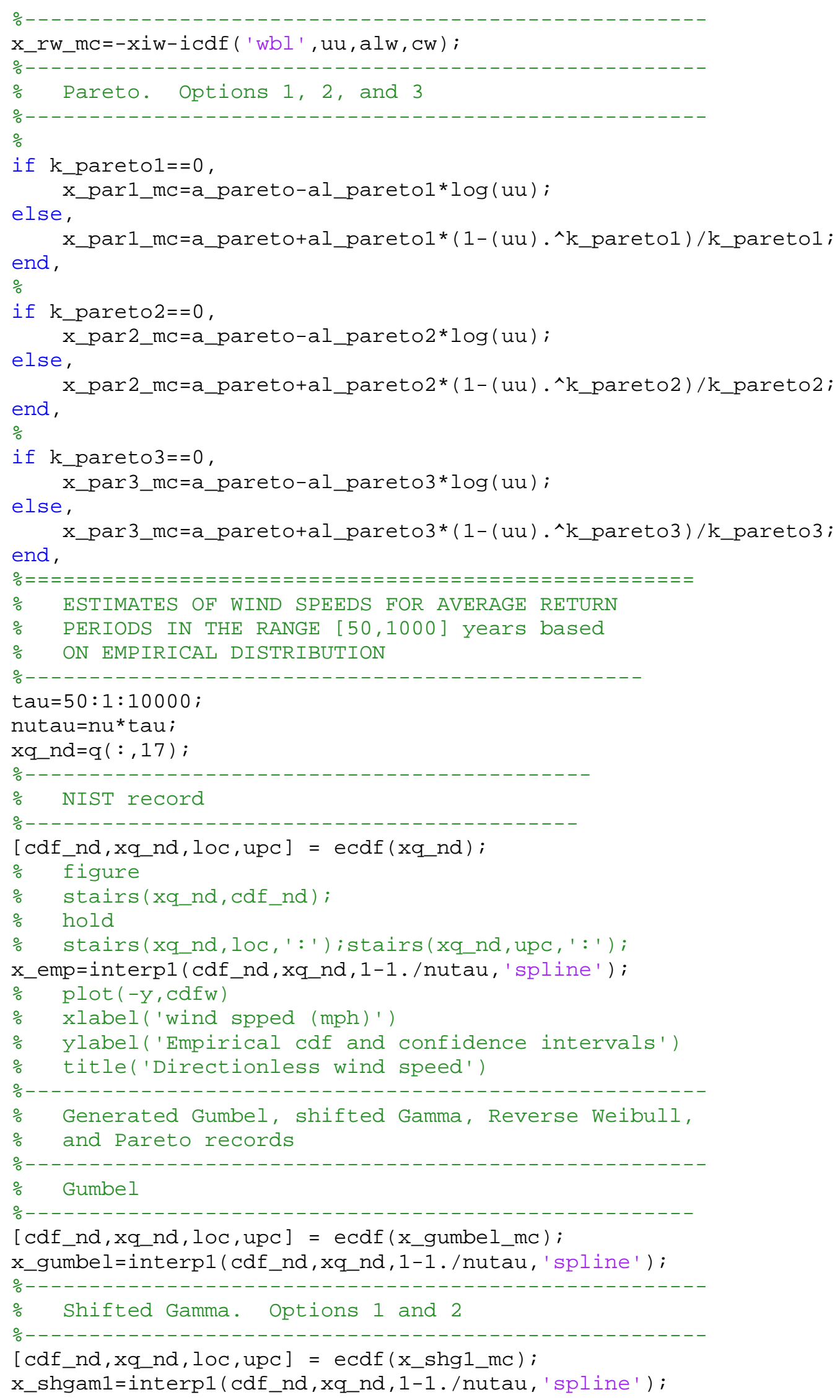


[cdf_nd, xq_nd, loc,upc] = ecdf (x_shg2_mc);

x_shgam2=interp1(cdf_nd, xq_nd,1-1./nutau, 'spline');

$\% \quad$ x_shgam1=shift1+icdf('Gamma',1-1./nutau, kq1,1/lamq1);

$\% \quad$ x_shgam2=shift2+icdf('Gamma', 1-1./nutau, kq2,1/lamq2);

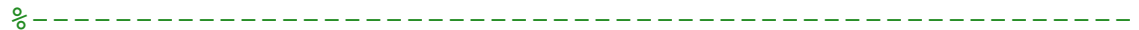

$\% \quad$ Reverse Weibull

$\%$ NOTE: Here the thinning consists in retaining

$\% \quad$ values smaller than a threshold

[cdf_nd, xq_nd, loc, upc] = ecdf $\left(x \_r w \_m c\right)$;

x_rw=interp1(cdf_nd, xq_nd, 1-1./nutau, 'spline' );

[alw cW xiw]

x_rw=-xiw-icdf( 'wbl', 1./nutau, alw, cw );

figure

plot(tau, x_shgam, tau, x_shgam2, tau, x_rw, ' : ' )

xlabel('Average return period (years)')

ylabel('Wind speed (mph)')

title('Gamma solid line; Reverse Weibull dotted line')

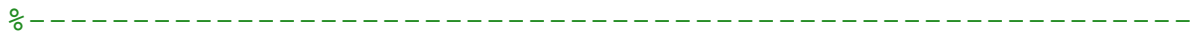

$\%$ Pareto. Options 1, 2, and 3

[cdf_nd, xq_nd, loc, upc] = ecdf (x_par1_mc);

x_par1=interp1(cdf_nd, xq_nd,1-1./nutau, 'spline' );

[cdf_nd, xq_nd, loc,upc] = ecdf (x_par2_mc);

x_par2=interp1(cdf_nd, xq_nd,1-1./nutau, 'spline' );

[cdf_nd, xq_nd, loc,upc] = ecdf(x_par3_mc);

x_par3=interp1(cdf_nd,xq_nd,1-1./nutau, 'spline' );

$\%$ if k_pareto1=-0,

\% x_par1=a_pareto-al_pareto1* $\log (1 . /$ nutau $)$;

$\%$ else,

$\%$ x_par1=a_pareto+al_pareto1*(1-(1./nutau).^k_pareto1)/k_pareto1;

\%end,

\%if k_pareto2==0,

$\%$ x_par2=a_pareto-al_pareto2* $\log (1 . /$ nutau $)$;

$\%$ else,

$\%$ x_par2=a_pareto+al_pareto2*(1-(1./nutau).^k_pareto2)/k_pareto2;

\%end,

\%if k_pareto3==0,

\% x_par3=a_pareto-al_pareto3* $\log (1 . /$ nutau $)$;

$\%$ else,

$\%$ x_par3=a_pareto+al_pareto3*(1-(1./nutau).^k_pareto3)/k_pareto3;

$\%$ end,

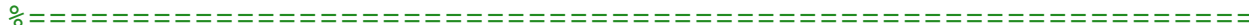

$\% \quad$ PLOT WIND SPEEDS WITH RETURN PERIODS OF $[50,1000]$ years

$\%$ DELIVERED BY SHIFTED GAMMA (X_shgam, $x$-shgam2),

$\%$ REVERSE WIBULL (x_rW), and PARETO (x_pareto) MODELS

$\% \quad \mathrm{X} \_$emp $=$ESTIMATE BASED ON DATA ONLY

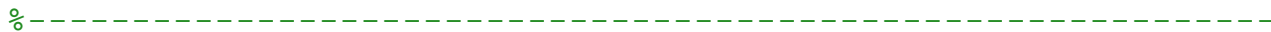

figure

plot (tau, x_gumbel, ' : ' , tau, x_rw, ' - - ',tau, x_par3, tau, x_emp, ' - . ')

xlabel('Average return period (years)')

ylabel('Wind speed (mph)')

title('Gumbel(dotted); Reverse Weibull(dashed); Pareto-DEHAAN (solid);

Empirical(dashdot)' )

figure

plot (tau, x_shgam1, 'o', tau,x_par1, ' - - ',tau,x_par2, ' : ', tau, x_par3, tau, x_emp, ' - .' ) 


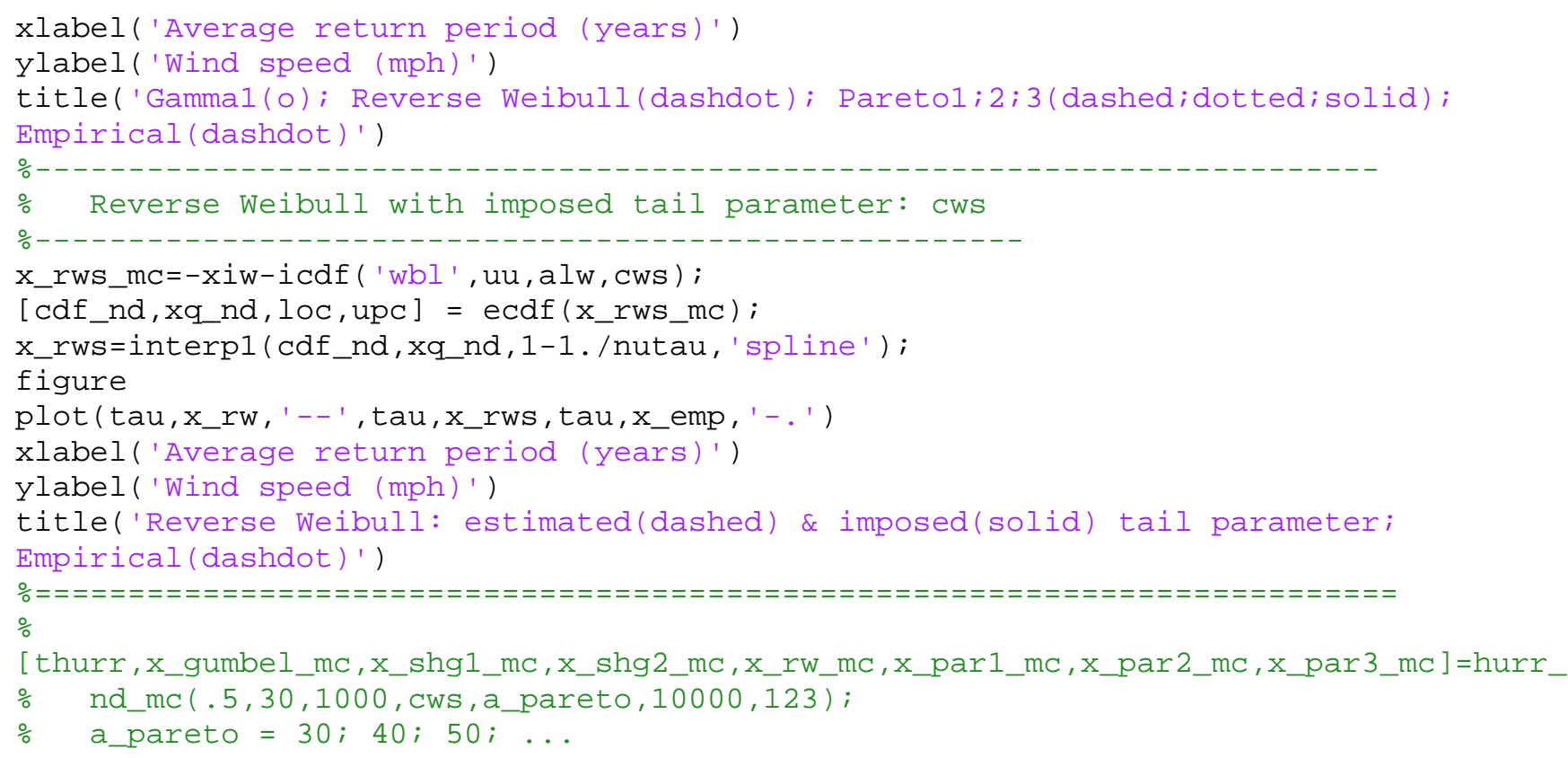

